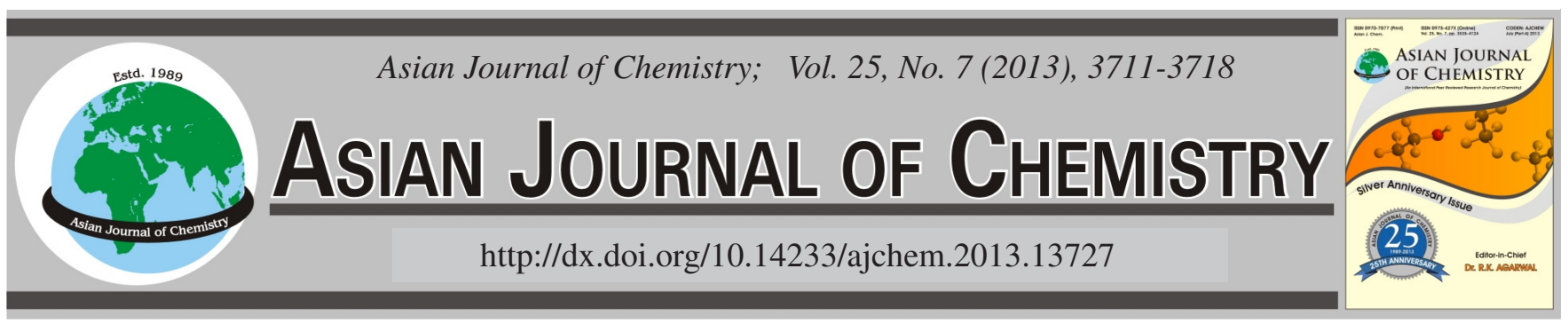

\title{
Molecularly Imprinted Membrane Possessing Selectivity and Adsorption Behaviour to Targeted (-)-Epigallocatechin-3-gallate Molecule
}

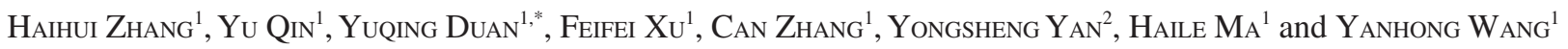

\author{
${ }^{1}$ School of Food and Biological Engineering, Jiangsu University, Zhenjiang 212013, P.R. China \\ ${ }^{2}$ School of Chemistry \& Chemical Engineering, Jiangsu University, Zhenjiang 212013, P.R. China \\ *Corresponding author: Fax: +86 511 88780201; Tel: +86 511 88780201; E-mail: dyq101@ujs.edu.cn
}

\begin{abstract}
An efficient molecular imprinted membrane (MIM) was prepared and successfully used for selective absorbing (-)-epigallocatechin-3gallate (EGCG) from the solution of tea. The MIM obtained was characterized by FT-IR and scanning electron microscopy. Kinetics, binding and selectivity experiment were used to study the adsorption properties of MIM in EGCG solution. The results showed that the template molecule had been incorporated into the support membrane by physical measure, the kinetics of MIM followed the pseudosecond-order kinetic model and the process of equilibrium adsorption could be simulated by Langmuir model. Molecular imprinted membrane exhibited better selectivity for EGCG from the extract of tea polyphenol than non-molecularly imprinted membrane (NIM) and the relative selectivity coefficients of MIM for EGCG/(-)-epigallocatechin, EGCG/(+)-catechin, EGCG/(-)-gallocatechin gallate and EGCG/(-)-epicatechin-3-gallate were 3.22, 1.34, 1.33 and 1.59, respectively. These findings indicated that the MIM had a good selectivity and could be used to separate EGCG from the real sample.
\end{abstract}

Key Words: Molecularly imprinted membrane, (-)-Epigallocatechin-3-gallate, Selectivity, Separation.

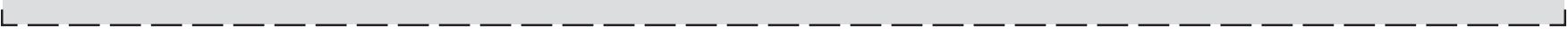

\section{INTRODUCTION}

(-)-Epigallocatechin-3-gallate (EGCG) is the major polyphenolic constituent found in green tea ${ }^{1}$. Several other polyphenolic compounds known as catechins are also found in lower abundance in green tea. These other catechins include (-)-epicatechin-3-gallate (ECG), (-)epigallocatechin (EGC), $(-)$ gallocatechin gallate (GCG) and (+)-catechin (C) and more than $50 \%$ of the catechin combination is composed of EGCG. Recent studies suggest that EGCG possesses certain biological activities, including antioxidant ${ }^{2-4}$, antimutagenic, anticarcinogenic and anti HIV activity ${ }^{5,6}$. In addition, EGCG is known to prevent dental caries and reduce the risk of cardio-vascular injury ${ }^{6,7}$. In recent years, because of these properties and applications, increased attention has been focused on the development of efficient separation and purification methods for EGCG. Methods that have been used previously for the separation of EGCG include preparative Sephadex LH-20 column chromatography ${ }^{8}$ and/or high-performance liquid chromatography ${ }^{9}$. All these methods require large amounts of solvent and plenty of time to obtain EGCG with high purity, therefore further study is still required.

Recently, molecular imprinting technology has been proven to introduce molecular recognition sites for a specific analyte in a synthetic polymer for selective separation or concentration of target molecules. This technique involves the formation of complexes between imprinting molecules (templates) and functional monomers/polymers based on the following three interactions: covalent bonds, non-covalent interactions or metal in coordinations ${ }^{10}$. Removal of the templates finally results in cavities with a shape, structure and functional groups complementary to the templates. It has been shown previously that molecularly imprinted polymers (MIPs) possesses high selectivity and sensitivity for low-molecularmass compound polymers, demonstrates very good thermal and mechanical stability and can be used in aggressive media ${ }^{11,12}$. Molecularly imprinted polymers have been widely studied and applied for chromatographic separation ${ }^{13,14}$, antibody mimics ${ }^{15}$ and selective elements of chemical sensors ${ }^{16}$, particularly in the solid-phase extraction (SPE) field ${ }^{17}$.

Introducing molecular recognition sites and allowing the specific recognition to a certain target molecule are regarded as the keys to the molecular imprinting technology ${ }^{18}$. The conventional way to obtain specific recognition sites in a polymeric matrix is to polymerize pre-organized solution of template, functional monomer, crosslinking agent and radical initiator, followed by grinding, sieving and template extraction. However, this approach has several shortcomings including 
time and labor cost, heterogeneity and damage of binding sites during the grinding step ${ }^{19,20}$.

In recent years, the imprinting technique has been developed to polymeric membranes, i.e., MIM, which can separate the targeted molecule from mixture solution by permeating through the thin membrane. MIM can be achieved by immobilizing the imprinting layer onto the substrate membrane without grinding ${ }^{21}$ or destroying the specific recognition sites during the grinding step. Besides the substrate can endow the imprinted membrane with robust and self-supporting properties. Molecular imprinted layer can be formed on the surface of the porous membrane with optimized flux via an interfacial polymerization technique ${ }^{22}$. In addition, the molecular imprinted sites can also be prepared inside a membrane via a phase inversion method in the presence of a template molecule ${ }^{23}$. The prepared membrane records the shape of the target template molecules during the phase inversion process. Consequently, MIM has potential applications in several fields and can be used as separation tool for its tailor-made design to the template molecule.

In the present study, we aim at developing a molecular imprint composite membrane with PVDF ultrafiltration membrane as the support for selective adsorption of EGCG. PVDF membrane is an ideal support for the imprinted membrane due to its excellent chemical and thermal stability, mechanical strength and filtration performance, furthermore, the porous structure of PVDF membrane with high flux optimized by a phase inversion method ${ }^{24}$ is in favour of the immobilization of imprinting layer.

To our knowledge, The usefulness of this work is to help understand the imprinting effect and recognition phenomenon. In addition this kind of imprinted polymeric membrane can be used as the selective absorbent or separation approaches for purification and provides foundation for appraisal of tea quality.

\section{EXPERIMENTAL}

EGCG, ECG, EGC, GCG and C (the purity of these reagents was $99 \%$ ) were supplied by the National Institute for the Control of Pharmaceutical and Biological Products (Beijing, China). (Chemical structures are shown in Fig. 1). Tea was purchased from Huangshan, (Anhui, China).

EDMA was supplied by Shanghai Shanhu Chemical Plant (Shanghai, China). Methacrylic acid (MAA), azobisisobutyronitrile $(\mathrm{AIBN})$, acetic acid and chloroform $(\mathrm{CHCl} 3)$ were purchased from Sinopharm Chemical Reagent Co. Ltd. (Shanghai, China). Methanol (HPLC grade) was purchased from Tedia (USA). EDMA and MAA were distilled under reduced operation pressure to remove the inhibitors whiles AIBN was re-crystallized with ethanol prior to use. All other chemicals were of analytical grade and used as received. PVDF was purchased from Shanghai Ofluorine Chemiacal Technology Co., Ltd (Shanghai, China). Deionized water was used throughout this study.

High performance liquid chromatography (Shimadzu, Japan) for determining EGCG compounds consisted of a binary LC-10ADvp pump, an DGU-12A on-line degasser, a CTO10Avp column oven, a SPD-10Avp UV-VIS detector and SCL10Avp system controller. The injection loop volume was $10 \mu \mathrm{L}$ and the analytical column was a $25 \mathrm{~cm} \times 0.46 \mathrm{~cm}$ i.d. spherical. The mobile phase consisted of Milli-Q-quality water, as solvent A (containing $0.3 \%, \mathrm{v} / \mathrm{v}$, acetic acid) and methanol as solvent B. Both solvent A and solvent B were mixed prior to reaching the analytical column. The flow rate of the mobile phase was $0.6 \mathrm{~mL} \mathrm{~min}{ }^{-1}$. The oven temperature was set at $38^{\circ} \mathrm{C}$ and the compounds were detected at $274 \mathrm{~nm}$.

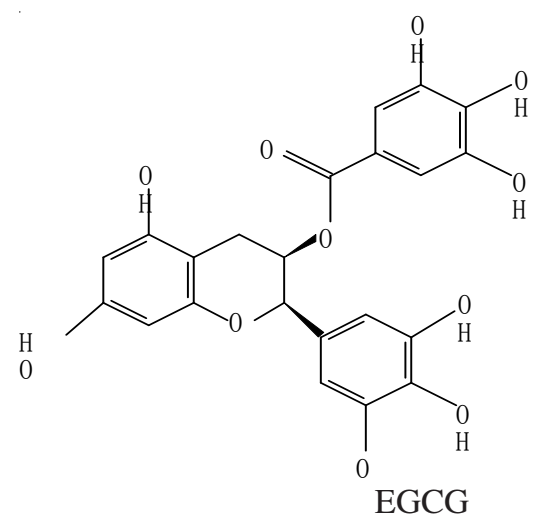<smiles></smiles><smiles></smiles>

ECG<smiles></smiles><smiles></smiles>

EGC

Fig. 1. Chemical structure of (-)-epicatechin-3-gallate (EGCG) and analogues 
The surface morphological images of prepared polymers were observed by S-4800 scanning electron microscope (Hitachi, Japan). Infra-red spectra were recorded in the range $4000-500 \mathrm{~cm}^{-1}$ using a Nicolet Nexus 470 FT-IR spectrometer (Thermo Fisher Scientific, USA). Analytical Balance (METTLER-Toledo Instruments (Shanghai Co., Ltd, China), UV-1601 (UV-VIS spectrophotometer), Centrifuge (Jintan, Jiangsu Ronghua Instrument Manufacturing Co., Ltd., China), ZF Ultrasonic Cleaner (Shanghai Zhi Feng Electronic Technology Co., Ltd., China), Constant temperature drying oven (Shanghai Experimental Instrument Factory Co., Ltd., China).

Preparation of MIM: In this study, molecular imprinted membrane was prepared using EGCG as template, MAA as functional monomer, EDMA as cross-linker and PVDF as support. A typical preparation of the molecular imprinted membrane was carried out following the protocol of ${ }^{25}$; moreover, the process of preparation was interpreted and shown by Fig. 2. To produce the imprinted polymer layer of MIM, PVDF membranes were coated in conical flask by soaking for $5 \mathrm{~min}$ in a $5 \mathrm{~mL}$ methanol and $3 \mathrm{~mL}$ chloroform solution containing $48.4 \mathrm{mg}$ EGCG and $34.4 \mathrm{mg}$ MAA, $0.4 \mathrm{~mL}$ EDMA and $8 \mathrm{mg}$ AIBN. Thereafter, membranes were placed in an oven and sparged with nitrogen gas $\left(\mathrm{N}_{2}\right)$ for $5 \mathrm{~min}$. Then, with the irradiation of $U V$ at $365 \mathrm{~nm}$ and environment of $\mathrm{N}_{2}$, the membranes were clamped between two quartz glasses and the polymerization reaction was carried out at room temperature for $10 \mathrm{~h}$ to obtain cross-linking structure.

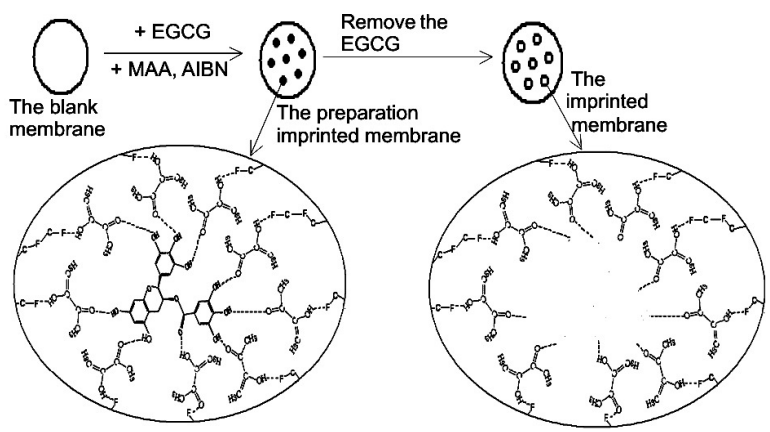

Fig. 2. Preparation of imprinted membrane for EGCG

The membranes were then extracted with methanol/ acetic acid $(9 / 1, v / v)$ for $3 \mathrm{~h}$ and washed with methanol to remove any non-grafted polymer, monomer, residual initiator and the template. The efficiency of this procedure was checked by the UV spectrum of the filtrate. The absorbance was $<0.005$ at $274 \mathrm{~nm}$.

The non-imprinted membranes (NIM) were also obtained with the same polymerization procedure without the template EGCG.

Characterization: The chemical structure of MIM was examined by FT-IR. The samples were placed on a sample holder (potassium bromide, $\mathrm{KBr}$ ) and all spectra were recorded in the wave number range of $4000-500 \mathrm{~cm}^{-1}$ by cumulating 32 scans at a resolution of $2 \mathrm{~cm}^{-1}$.

The morphological structures of MIM were examined by SEM. The cross section of the membrane was obtained by the fracture of the membrane in liquid nitrogen. Both the surface and cross section of the samples were sputtered by gold for $25 \mathrm{~s}$ before observation.
Binding experiments: Static binding experiments were conducted to evaluate the recognition property of the membranes toward the target molecule. Concentrations of 10, 20, $30,40,50,60,70$ and $80 \mu \mathrm{gL}^{-1}$ of EGCG in methanol were prepared. Binding experiments were carried out in a conical flask containing about $0.5 \mathrm{mg}$ of MIM and $5 \mathrm{~mL}$ of the test solution (each concentration mentioned above). The conical flask was shaken for $9 \mathrm{~h}$ in a methanol bath at $30^{\circ} \mathrm{C}$. The residual EGCG in the supernatant was measured by UV spectrometry at $274 \mathrm{~nm}$.

The binding characteristics of MIM for EGCG were determined throughout the experiments (each chemical binding experiment was replicated three times and the mean value was calculated). The mean value was used to calculate the binding capacity of the MIM using the eqn. 1:

$$
\mathrm{Q}=\left(\mathrm{C}_{0}-\mathrm{C}_{\mathrm{e}}\right) \times \frac{\mathrm{V}}{\mathrm{m}}
$$

where, $\mathrm{Q}\left(\mathrm{mg} \mathrm{g}^{-1}\right)$ represents the binding capacity of the MIM for the solute (EGCG); $\mathrm{C}_{0}$ and $\mathrm{C}_{\mathrm{e}}\left(\mu \mathrm{g} \mathrm{mL} \mathrm{m}^{-1}\right)$ represent the feed concentration at the initial time and the equilibrated binding time, respectively; $\mathrm{V}$ represents the volume of feed solution $(\mathrm{mL})$; $\mathrm{m}$ represents the weight of MIM or NIM (mg).

In order to evaluate the selectivity of the sorbents, the static distribution coefficient $\mathrm{K}_{\mathrm{D}}$ is used to express the specific adsorption of MIM. In addition, the selectivity coefficient for rebinding a EGCG molecule coexisted in competitive species can be obtained from equilibrium binding data. Thus $K_{D}$ and $\mathrm{K}$ are defined by eqns. 2 and 3 , respectively ${ }^{26}$ :

$$
\begin{gathered}
\mathrm{K}_{\mathrm{D}}=\frac{\mathrm{Q}_{\mathrm{e}}}{\mathrm{C}_{\mathrm{e}}} \\
\mathrm{K}=\frac{\mathrm{K}_{\mathrm{D}(\mathrm{EGCG})}}{\mathrm{K}_{\mathrm{D}(\mathrm{x})}}
\end{gathered}
$$

where $K_{D}$ is the distribution coefficient, $Q_{e}$ is the equilibrium adsorption capacity $\left(\mathrm{mg} \mathrm{g}^{-1}\right) ; \mathrm{C}_{\mathrm{e}}$ is the equilibrium concentration $\left(\mu \mathrm{g} \mathrm{mL}^{-1}\right) . \mathrm{K}$ is the selectivity coefficient and represents EGCG molecular adsorption selectivity when there are other analogs in solution. $\mathrm{x}$ is EGC, C, ECG or GCG molecule.

$\mathrm{K}^{\prime}$ is a relative selectivity coefficient and represents the difference in EGCG adsorption affinity recognition of sites to the imprinted EGCG molecules between them. K' can be expressed by eqn. 4 :

$$
\mathrm{K}^{\prime}=\frac{\mathrm{K}_{(\mathrm{MIM})}}{\mathrm{K}_{(\mathrm{NIM})}}
$$

where $\mathrm{K}_{\text {(MIM) }}$ and $\mathrm{K}_{(\mathrm{NIM})}$ are the selectivity coefficients of the MIM and NIM, respectively.

Adsorption kinetics: The uptake kinetics of EGCG by MIM was also examined. The MIM $(0.5 \mathrm{mg})$ was added to 5 $\mathrm{mL}$ of EGCG $\left(40 \mu \mathrm{g} \mathrm{mL}^{-1}\right)$ methanol solution. The mixture was mechanically shaken (200 times $\left.\mathrm{min}^{-1}\right)$ for $0.5,1,1.5,2$, $2.5,3,4,5,6,8$ and $9 \mathrm{~h}$ at $30^{\circ} \mathrm{C}$. The residual EGCG in the supernatant was measured by UV spectrometry at $274 \mathrm{~nm}$.

\section{RESULTS AND DISCUSSION}

FT-IR characterization: The FT-IR spectra of support membrane, non-imprinted composite membrane with template 
and imprinted membrane with template were presented in Fig. 3(A-C). As shown in Fig. 3A, there were no obvious absorption bands between 2980 and $2950 \mathrm{~cm}^{-1}$, which indicated no $-\mathrm{CH}_{3}$ in the PVDF polymer. Fig. $3 \mathrm{C}$ exhibited that the strong absorption peak of $3028 \mathrm{~cm}^{-1}$ may correspond with the absorption bands of -C-H stretching vibration. The 1461, 1542 and $1643 \mathrm{~cm}^{-1}$ indicated $-\mathrm{C}=\mathrm{C}$ - stretching vibration whereas the four absorption peaks showed the appearance of a benzene ring. The peak at $693 \mathrm{~cm}^{-1}$ was assignable to the interdisubstitution in the benzene ring. Another absorption peak near $2929 \mathrm{~cm}^{-1}$ contributed to the stretching vibration of $-\mathrm{CH}_{2}$. It can be seen that the shape and position of all peaks in Fig. 3B and $\mathrm{C}$ were very similar, signifying that both imprinted and non-imprinted membranes had the same chemical composition of the modified layer. These behaviour demonstrated that the template molecule had been incorporated into the support membrane before being extracted.

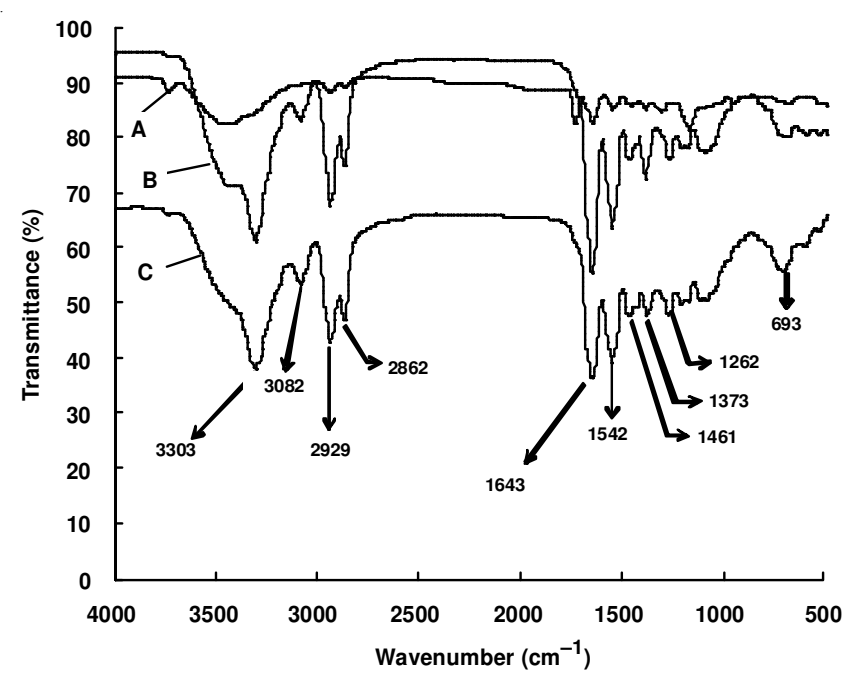

Fig. 3. FT-IR spectra of (A) support membrane, (B) imprinted membrane with template, $(\mathrm{C})$ non-imprinted membrane with template

Morphology characterization: The surface morphology of the resultant polymer membranes with the support of cellulosic membrane was observed with SEM. Fig. 4 showed the SEM images of the PVDF (A and C) and imprinted membrane (B and D). The SEM images (Fig. 4A and C) confirmed the dense structure of the membranes prepared. By comparing A and $\mathrm{B}$ (magnification 2,000) the loose membrane gap was observed and this kind of membrane gap provided a good place for combination template (EGCG) and functional monomer (MAA). When the magnification was 10,000, it was observed that the bright spots adhered to the membrane gap, revealing that the cross-linker played its role successfully.

Binding isotherms of MIM: Fig. 5A showed the adsorption characterization of MIM and NIM with the concentration of EGCG increasing from $0-80 \mu \mathrm{gL}^{-1}$. It is clear how adsorption capacity increased with EGCG concentration to an equilibrium of $70 \mu \mathrm{g} \mathrm{mL}^{-1}$. The greatest difference of 2.9889 $\mathrm{mg} \mathrm{g}^{-1}$ was observed between MIM and NIM when the adsorption equilibrium was reached.

Adsorption isotherms are important for describing how adsorbates will interact with adsorbent ${ }^{27}$. Thus, the correlation of equilibrium data using either a theoretical or empirical
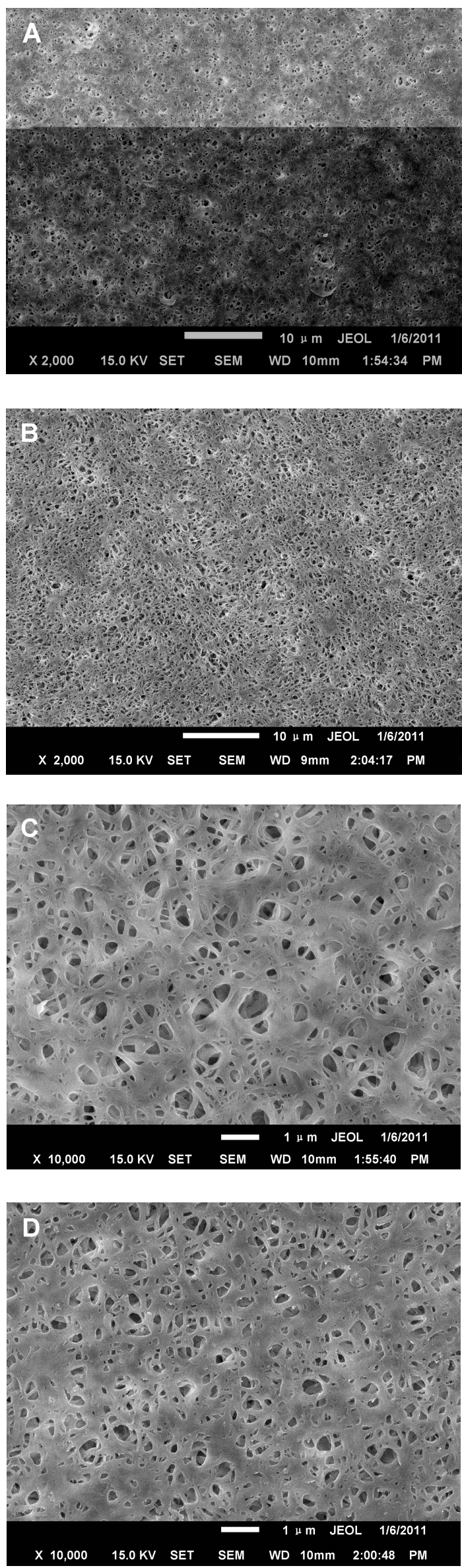

Fig. 4. SEM images of the PVDF membrane (A and $\mathrm{C}$ ) and imprinted composite membrane (B and D) 


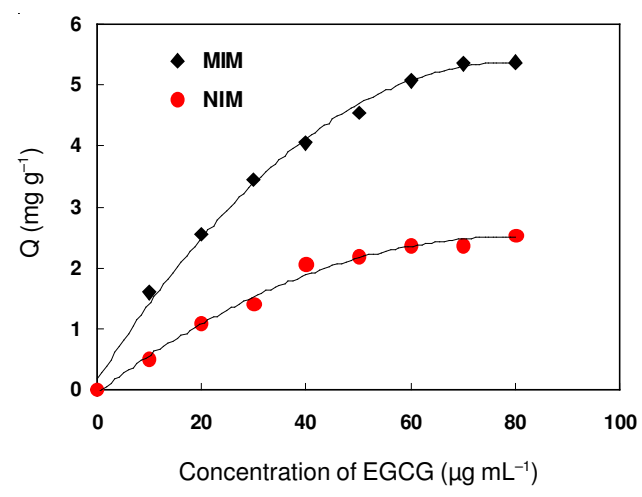

(A)

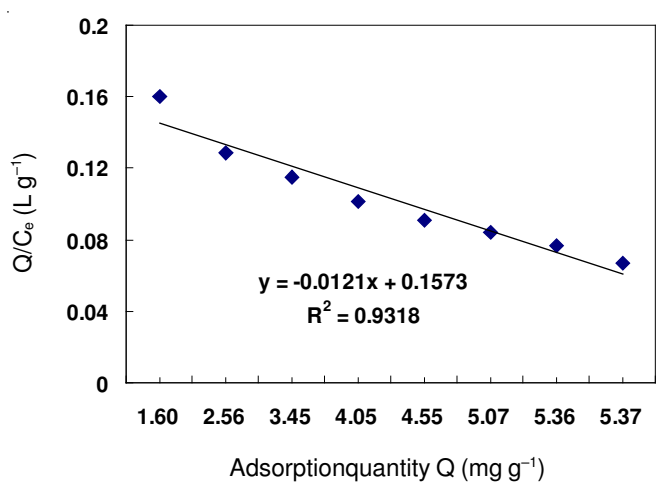

(B)

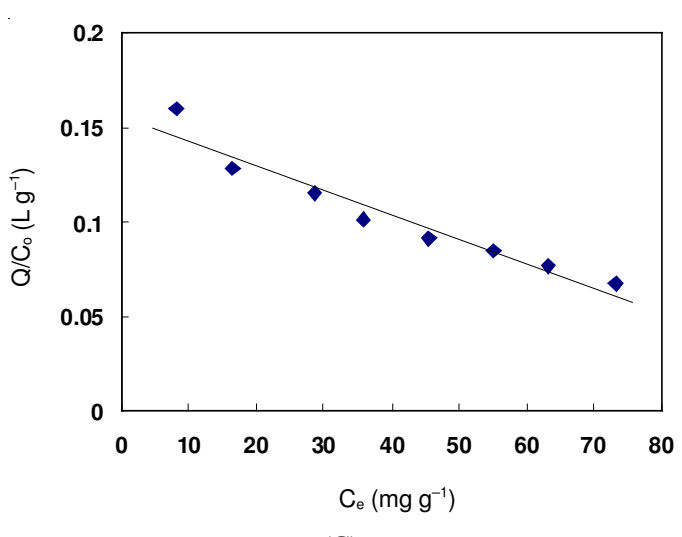

(C)

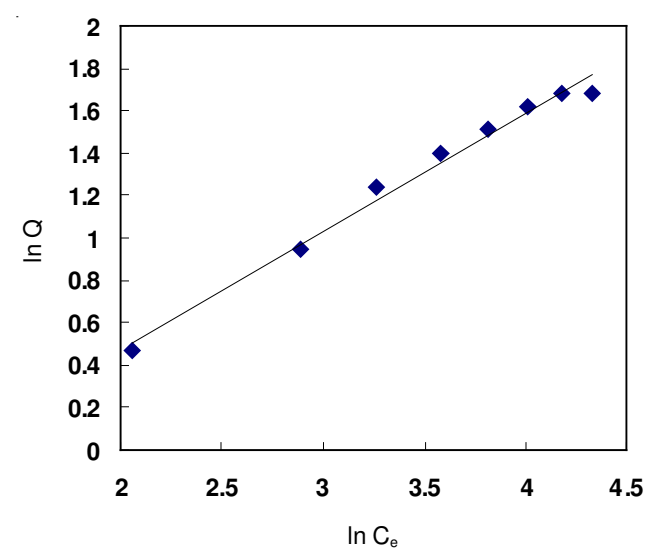

(D)

Fig. 5. Study on the static adsorption characteristics of MIM. Binding isotherm of polymer (A), Scatchard plots (B), Langmuir linear plot (C) and Freundlich model (D). $\mathrm{C}_{0}$ of EGCG was $0-80 \mu \mathrm{g} \mathrm{mL} \mathrm{m}^{-1}$, time $=9 \mathrm{~h}$; volume $=5 \mathrm{~mL} ;$ mass $=0.5 \mathrm{mg}$; temperature $=30^{\circ} \mathrm{C}$ equation is essential to practical adsorption operation. Three isotherm equations were used in the present study i.e., Scatchard model, Langmuir model and the Freundlich model ${ }^{28}$.

Scatchard model was used here to evaluate the binding characteristics of molecularly imprinted polymers, The Scatchard model analysis formula (eqn. 5) is:

$$
\frac{\mathrm{Q}}{\mathrm{C}_{\mathrm{e}}}=\frac{\left(\mathrm{Q}_{\max }-\mathrm{Q}\right)}{\mathrm{K}_{\mathrm{d}}}
$$

where $\mathrm{Q}$ and $\mathrm{Q}_{\max }$ represent the apparent equilibrium binding capacity $\left(\mathrm{mg} \mathrm{g}^{-1}\right)$ and the maximum binding capacity $\left(\mathrm{mg} \mathrm{g}^{-1}\right)$, respectively, $\mathrm{C}_{\mathrm{e}}$ is the equilibrium concentration of the solution $\left(\mu \mathrm{g} \mathrm{mL}^{-1}\right.$ ) and $\mathrm{K}_{\mathrm{d}}$ is the binding sites of the equilibrium dissociation constant. The $\mathrm{Q} / \mathrm{C}_{\mathrm{e}}$ associates with $\mathrm{Q}$ mapping in accordance with the linear relationship between the slope and intercept, which can be obtained using the two parameters $\mathrm{K}_{\mathrm{d}}$ and $\mathrm{Q}_{\max }$. A straight line was available by mapping of $\mathrm{Q} / \mathrm{C}_{\mathrm{e}}$ to $\mathrm{Q}$, as shown in Fig. 5B. The obtained equation was $\mathrm{Q} / \mathrm{C}_{\mathrm{e}}=$ $-0.0121 \mathrm{Q}+0.1573$, with the correlation coefficient $\mathrm{r}$ of 0.9318 . From the slope and intercept, $\mathrm{K}_{\mathrm{d}}$ and $\mathrm{Q}_{\max }$ were calculated to be 82.64 and $13.00 \mathrm{mg} \mathrm{g}^{-1}$, respectively.

Langmuir model (eqn. 6):

$$
\frac{\mathrm{C}_{\mathrm{e}}}{\mathrm{Q}}=\frac{\mathrm{C}_{\mathrm{e}}}{\mathrm{Q}_{\max }}-\frac{1}{\mathrm{bQ}_{\max }}
$$

where $\mathrm{C}_{\mathrm{e}}$ is the final equilibrium concentration of EGCG $(\mu \mathrm{g}$ $\left.\mathrm{mL}^{-1}\right), \mathrm{Q}_{\mathrm{e}}$ is the adsorption capacity of EGCG adsorbed per unit weight of MIM at equilibrium concentration $\left(\mathrm{mg} \mathrm{g}^{-1}\right), \mathrm{Q}_{\max }$ is the maximum adsorption capacity $\left(\mathrm{mg} \mathrm{g}^{-1}\right)$, $\mathrm{b}$ is the Langmuir adsorption equilibrium constant $\left(\mathrm{g} \mathrm{L}^{-1}\right)$. The Langmuir regression equation obtained was $\mathrm{C}_{\mathrm{e}} / \mathrm{Q}_{\mathrm{e}}=0.1259 \mathrm{Ce}+4.2492$, the rearranged form of Langmuir adsorption equation: $\mathrm{Q}_{\mathrm{e}}=$ $\left(\mathrm{Q}_{\max } \mathrm{bC}_{\mathrm{e}}\right) /\left(1+\mathrm{bC}_{\mathrm{e}}\right)$ was $\mathrm{C}_{\mathrm{e}} / \mathrm{Q}_{\mathrm{e}}=\mathrm{C}_{\mathrm{e}} / \mathrm{Q}_{\max }+1 / \mathrm{bQ}_{\max }$. The plot of $\mathrm{C}_{\mathrm{e}} / \mathrm{Q}_{\mathrm{e}}$ versus $\mathrm{C}_{\mathrm{e}}$ is displayed in Fig. $5 \mathrm{C}$ with the correlation coefficient $r$ of 0.9901 . This indicated that the Langmuir plot was a line, the slope and intercept of which were equal to $\mathrm{Q}_{\max }$ and $1 / b Q_{\max }$, respectively and the binding sites formed due to template effect in the imprinting process in MIM were homogeneous with respect to the affinity for EGCG and the nonspecific adsorption to MIM could be assumed to be small enough to be ignored in this concentration range. The values of $\mathrm{b}$ and $\mathrm{Q}_{\max }$ were respectively 1.8692 and $7.9428 \mathrm{mg} \mathrm{g}^{-1}$, which can be calculated from the slope and the intercept of the Langmuir regression equation.

The Langmuir dimensionless constant separation factor, $\mathrm{R}_{\mathrm{L}}$, was also calculated according to the dimensionless expression, $\mathrm{R}_{\mathrm{L}}=1 /\left(1+\mathrm{bC}_{0}\right)$, where $\mathrm{C}_{0}$ is the initial concentration of EGCG. $\mathrm{R}_{\mathrm{L}}$ is commonly used as an indicator in analyzing the adsorption isotherms which can be classified into four types, unfavorable, linear, favorable and irreversible, respectively corresponding to the following four different cases, $R_{L}$ $>1, R_{L}=1,0<R_{L}<1$ and $R_{L}=1^{29}$. All the $R_{L}$ values, decreasing from 0.7716-0.2969 while the initial EGCG concentration increased from $10-80 \mu \mathrm{g} \mathrm{mL}^{-1}$, were lower than 1 , revealing a favorable adsorption of EGCG on the MIM.

Freundlich model (eqn. 7)

$$
\ln \mathrm{Q}=\ln \mathrm{Q}_{\mathrm{r}}+\frac{1}{\mathrm{n}}\left(\ln \mathrm{C}_{\mathrm{e}}\right)
$$


where $\mathrm{Q}_{\mathrm{f}}$ is roughly an indicator of the adsorption capacity $\left(\mathrm{mg} \mathrm{g}^{-1}\right)$ and $\mathrm{n}$ is the heterogeneity index, between 0 and 1 and strongly heterogeneous surfaces have small values of $n$ while less heterogeneous ones have values closer to unity. The adsorption energy distribution corresponding to the Freundlich model is an exponential decay. The Freundlich regression equation obtained was $\ln \mathrm{Q}=0.5554 \mathrm{C}_{\mathrm{e}}-0.6336$. This was a In $\mathrm{Q}$ versus $\ln \mathrm{C}_{\mathrm{e}}$ equation with the correlation coefficient $\mathrm{r}$ of 0.9866 (Fig. 5D). The $\mathrm{n}$ and $\mathrm{Q}_{\mathrm{f}}$ values were calculated to be 1.8692 and $9.3664 \mathrm{mg} \mathrm{g}^{-1}$ from the slope and the intercept of the Freundlich regression equation, respectively. The value $n$ $=1.8005(>1)$ again confirmed the favorable adsorption behavior of the imprinted hybrid membrane. The above Scatchard analysis agreed with the results of Langmuir and Freundlich fitting where the Langmuir model fitted relatively better than the Freundlich model. Therefore, the process of equilibrium adsorption can be simulated by Langmuir model.

Adsorption kinetics of MIM: It is well known that the adsorption kinetics can provide a help for illustrating the efficiency of adsorption and controlling the residual time of the whole adsorption process. The kinetics of EGCG adsorption was investigated by changing the enrichment time from $0-9 \mathrm{~h}$ under the adsorption of MIM by settled concentration of EGCG simultaneously. Fig. 6A shows the amount of EGCG adsorbed on the MIM as a function of time. High adsorption rates were observed at the beginning of adsorption and saturation values were reached within $4 \mathrm{~h}$. Around $4 \mathrm{~h}$ the curve became flat, meaning the adsorption reached equilibrium.

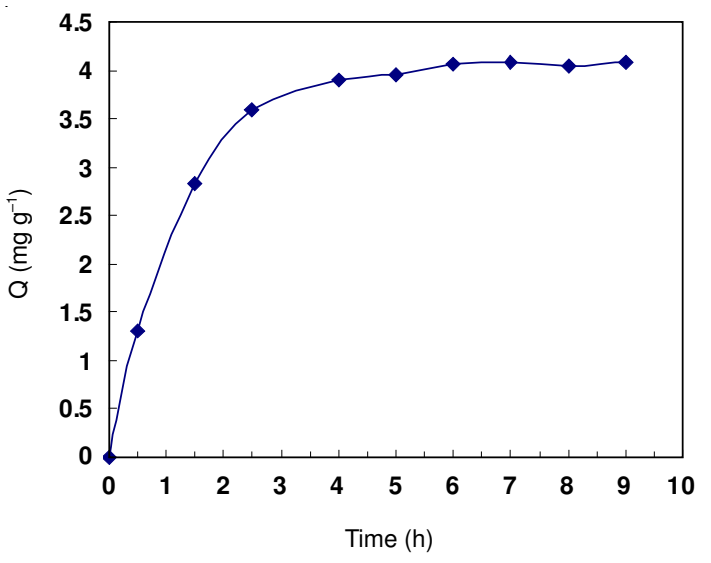

(A)

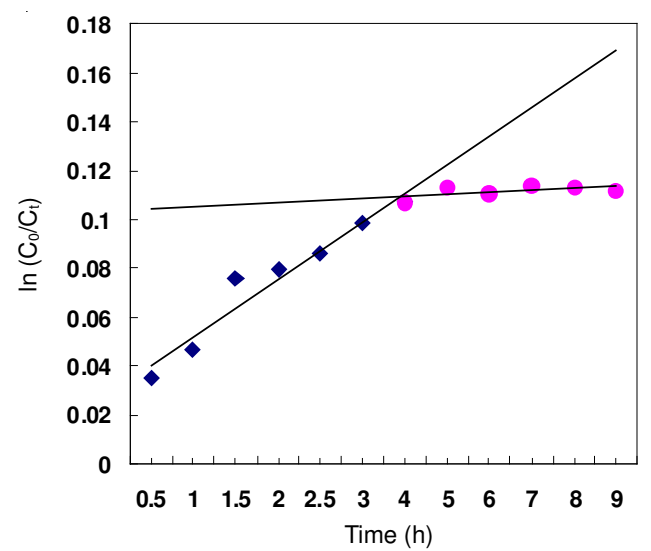

(B)

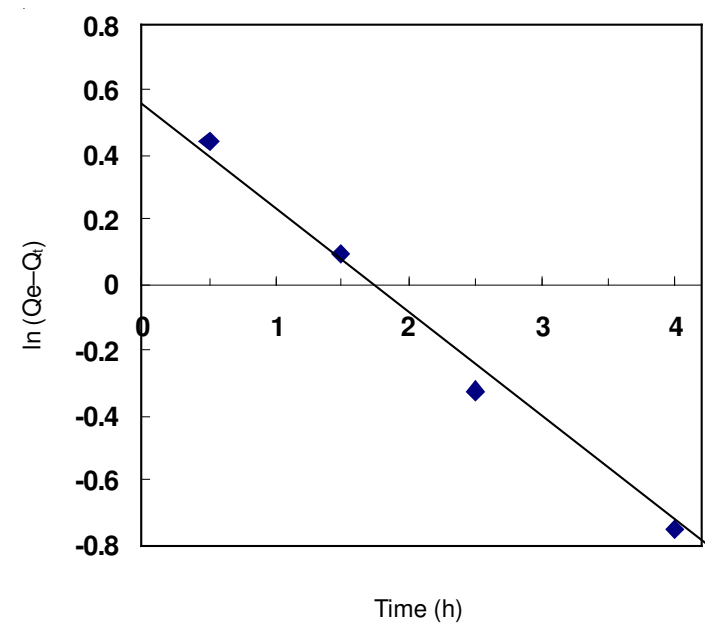

(C)

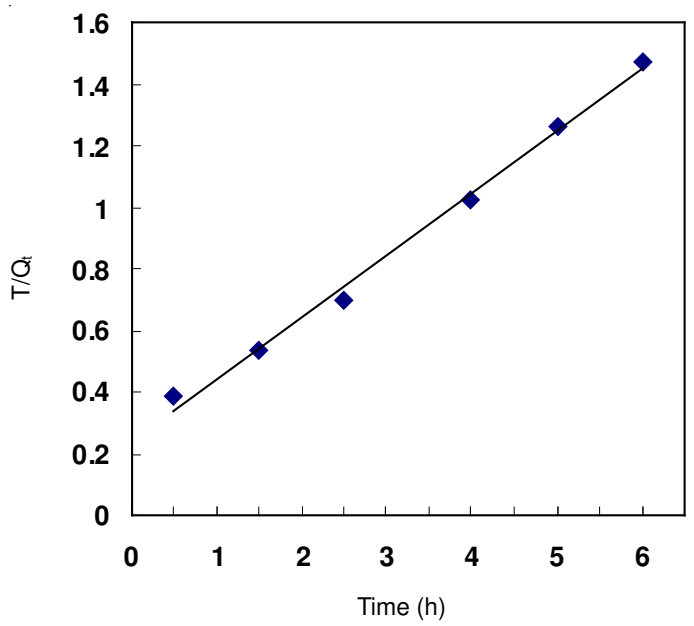

(D)

Fig. 6. Study on the adsorption dynamic characteristics of molecularly imprinted membrane (MIM). Adsorption dynamic curves (A) and $\ln \left(\mathrm{C}_{0} / \mathrm{C}\right)$ and $\mathrm{t}$ dynamic curves (B), pseudo-first-order $(\mathrm{C})$ and

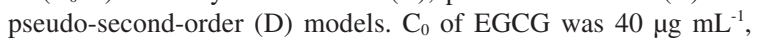
time $=9 \mathrm{~h}$; volume $=5 \mathrm{~mL}$; mass $=0.5 \mathrm{mg}$; temperature $=30^{\circ} \mathrm{C}$

Before $9 \mathrm{~h}$, as shown in Fig. 6B, we gave the linear relationship of $\ln \left(\mathrm{C}_{0} / \mathrm{C}\right)$ and $\mathrm{t}$. The first line illustrated that EGCG initially adsorbed on the surface of MIM with a fast adsorption rate before $4 \mathrm{~h}$. Compared with the first line, the second straight line had a slightly upward trend, indicating the absorption rate became slower, which demonstrated that it was much more difficult for EGCG to transfer from the surface of MIM into the internal and therefore absorption could be close to balance.

In order to analyze the adsorption of EGCG onto the MIM, the pseudo-first-order (eqn. 8) and pseudo-second-order (eqn. 9) models were given as the following equations ${ }^{30}$ :

$$
\begin{gathered}
\ln \left(\mathrm{Q}_{\mathrm{e}}-\mathrm{Q}_{\mathrm{t}}\right)=\ln \mathrm{Q}_{\mathrm{e}}-\mathrm{k}_{1} \mathrm{t} \\
\frac{\mathrm{t}}{\mathrm{Q}_{\mathrm{t}}}=\frac{1}{\left(\mathrm{k}_{2} \mathrm{Q}_{\mathrm{e}}^{2}\right)}+\frac{\mathrm{t}}{\mathrm{Q}_{\mathrm{e}}}
\end{gathered}
$$

where $\mathrm{Q}_{\mathrm{e}}$ and $\mathrm{Q}_{\mathrm{t}}$ are the amount of EGCG adsorbed $\left(\mathrm{mg} \mathrm{g}^{-1}\right)$ on the MIM at the equilibrium and at time $\mathrm{t}$, respectively, $\mathrm{k}_{1}$ $\left(\mathrm{h}^{-1}\right)$ and $\mathrm{k}_{2}\left(\mathrm{~h} \mathrm{~g} \mathrm{mg}^{-1}\right)$ are the rate constants of adsorption. The 
value of $\mathrm{k}_{1}$ was calculated from the plots of $\ln \left(\mathrm{Q}_{\mathrm{e}}-\mathrm{Q}_{\mathrm{t}}\right)$ versus $\mathrm{t}$ by pseudo-first-order and $\mathrm{k}_{2}$ was obtained from plotting $\left(t / Q_{t}\right)$ versus $t$ by the pseudo-second-order.

Comparing the fitting results of the models, the adsorption of EGCG onto MIM could be best described by the pseudo-first-order rate equation. As shown in Fig. 6C and D, the pseudo-first-order rate equation obtained was $\ln \left(\mathrm{Q}_{\mathrm{e}}-\mathrm{Q}_{\mathrm{t}}\right)$ $=-0.3186 t+0.5562$, with the correlation coefficient $\mathrm{r}$ of 0.9989 and the $\mathrm{k}_{1}$ of 0.7337 Error! Reference source not found. The pseudo-second-order rate equation obtained was $t / Q_{t}=0.2027$ $t+0.2362$ Error! Reference source not found., with the correlation coefficient $r$ of 0.9942 and the $\mathrm{k}_{2}$ of 0.1740 Error! Reference source not found.. It was demonstrated that the fit of the experimental data by the pseudo-first-order kinetic model gave higher correlation coefficients from the two models, the corresponding correlation coefficients and the rate contant of adsorption. Therefore, the adsorption behaviour of EGCG onto MIM followed the pseudo-first-order kinetic model, indicating that the adsorption process was chemical in nature.

Selectivity of MIM: In order to investigate the recognition ability of MIM, four different kinds of analogue were used for the target/competitive substance (i.e., adsorption from solution containing $40 \mu \mathrm{g} \mathrm{mL} \mathrm{m}^{-1}$ of EGCG and its analogue). Both the MIM and NIM had adsorption amount for several substances but the adsorption capacity for MIM was more than for NIM (except for EGC) and a similar phenomenon also occurred in the MIM for EGCG and its analogue (Table-1). When they exist in the same medium, a competition will start for the same attachment sites.

In addition, the distribution and selectivity coefficient $\left(\mathrm{K}_{\mathrm{d}}\right)$, selectivity coefficient $(\mathrm{k})$ and relative selectivity coefficient $\left(\mathrm{k}^{\prime}\right)$ can usually be used to estimate the adsorption selectivity
(Table-1). A comparison of the selectivity coefficient of MIM with the selectivity coefficient of NIM showed that the MIM for EGCG/EGC, EGCG/C, EGCG/GCG and EGCG/ECG were $3.2230,1.3425,1.3292$ and 1.5880 greater than NIM. It can be concluded that the MIM showed the following affinity order under competitive conditions: EGCG > GCG > C > ECG > EGC. This meant that EGCG could be determined even in the presence of EGC, C, GCG and ECG interferences and after removal of templates the imprinting sites with "memory" created and showed the selectivity of binding ability.

Real sample: The accuracy of the method was estimated by adsorbing the amount of EGCG in the initial extraction of tea polyphenol solution. In this experiment, the method for water extraction of tea polyphenol (TP) from green tea followed by Li et al. ${ }^{31}$. Under this condition, tea polyphenol was extracted successfully including several kinds of monomers using $200 \mu \mathrm{g} \mathrm{mL}^{-1}$ of tea polyphenol extraction for the adsorption solution. Several kinds of monomers could be detected by HPLC in the solution (Table-2).

After absorption for $4 \mathrm{~h}$, the results were determined by HPLC at $274 \mathrm{~nm}$ and it was shown in Fig. 7(A-C). Fig. 7(B and $\mathrm{C}$ ) showed that several kinds of monomers were detected by HPLC after the initial tea extraction was absorbed by MIM and NIM respectively (several kinds of monomers in the initial tea extraction were showed by Fig. 7A). As could be observed from the MIM (Fig. 7B), after absorption, the concentration of EGCG was low enough to be quantitatively analyzed while it was high to be quantitated without absorption (Fig. 7A). However,the NIM had no obvious difference (Fig. 7A and C). Meanwhile, owing to the selectivity of MIM, it had no evident difference that the amounts for its analogue were absorbed by MIM and NIM.

TABLE-1

CALIBRATION DATA AND COMPETITIVE LOADING OF (-)-EPIGALLOCATECHIN GALLATE (EGCG) AND ANALOGUES BY MOLECULARLY IMPRINTED MEMBRANE (MIM) AND NON-MOLECULARLY IMPRINTED MEMBRANE (NIM)

\begin{tabular}{lccccc}
\hline \multicolumn{1}{c}{ Polymer and calibration data } & EGCG & (EGC) & $(\mathrm{C})$ & $(\mathrm{GCG})$ & $(\mathrm{ECG})$ \\
\hline Concentration and peak area & $\mathrm{y}=10.638 \mathrm{x}-35.23$ & $\mathrm{y}=3.5901 \mathrm{x}-10.55$ & $\mathrm{y}=6.5338 \mathrm{x}-9.76$ & $\mathrm{y}=8.3011 \mathrm{x}-90.41$ & $\mathrm{y}=10.65 \mathrm{x}-80.15$ \\
regression equation & & & & & \\
Correlation & $\mathrm{r}=0.9997$ & $\mathrm{r}=0.9994$ & $\mathrm{r}=0.9998$ & $\mathrm{r}=0.9999$ & $\mathrm{r}=0.9997$ \\
Linear range $\left(\mu \mathrm{g} \mathrm{mL}^{-1}\right)$ & $0-200$ & $0-200$ & $0-200$ & $0-200$ & $0-200$ \\
MIM adsorption capacity $\left(\mathrm{mg} \mathrm{g}^{-1}\right)$ & 4.01 & 1.54 & 2.4 & 2.84 & 1.78 \\
$\mathrm{NIM}$ adsorption capacity $\left(\mathrm{mg} \mathrm{g}^{-1}\right)$ & 1.95 & 2.23 & 1.51 & 1.78 & 1.31 \\
${ }^{\mathrm{a}} \mathrm{K}_{\mathrm{d} \text { MIM }}$ & 0.11 & 0.04 & 0.06 & 0.08 & 0.05 \\
${ }^{\mathrm{a}} \mathrm{K}_{\mathrm{d} \text { NIM }}$ & 0.05 & 0.06 & 0.04 & 0.05 & 0.03 \\
${ }^{b} \mathrm{k}_{\text {MIM }}$ & & 2.79 & 1.75 & 1.45 & 2.40 \\
${ }^{b} \mathrm{k}_{\mathrm{NIM}}$ & & 0.87 & 1.31 & 1.09 & 1.51 \\
${ }^{\mathrm{c}} \mathrm{k}$ ' & 3.22 & 1.34 & 1.33 & 1.59 \\
\hline
\end{tabular}

$\mathrm{K}_{\mathrm{d}}$, distribution coefficient; $\mathrm{K}_{\mathrm{d}}=\mathrm{Q}_{\mathrm{e}} / \mathrm{C}_{\mathrm{e}}$, where $\mathrm{Q}_{\mathrm{e}}$ and $\mathrm{C}_{\mathrm{e}}$ represent the equilibrium binding quantity and equilibrium concentration. ${ }^{\mathrm{b}} \mathrm{k}$ : selectivity coefficient; $\mathrm{k}=\mathrm{K}_{\mathrm{d} \text { EGCG }} / \mathrm{K}_{\mathrm{d} \text { analogues }}$; ${ }^{\mathrm{k}} \mathrm{k}$ ': relative selectivity coefficient, $\mathrm{k}^{\prime}=\mathrm{k}_{\text {MIMs }} / \mathrm{k}_{\text {NIMs }}$.

TABLE-2

ADSORPTION CHARACTER OF MOLECULARLY IMPRINTED MEMBRANE (MIM) AND NON-MOLECULARLY IMPRINTED MEMBRANE (NIM) IN REAL SAMPLE

\begin{tabular}{|c|c|c|c|c|c|c|c|}
\hline \multirow{2}{*}{ Sample } & \multirow{2}{*}{$\begin{array}{c}\mathrm{C}_{\mathrm{s}} \\
\left(\mu \mathrm{mL}^{-1}\right)\end{array}$} & \multirow{2}{*}{$\begin{array}{c}\text { Main } \\
\text { monomer }\end{array}$} & \multirow{2}{*}{$\begin{array}{c}\mathrm{C}_{\mathrm{o}} \\
\left(\mu \mathrm{mL}^{-1}\right)\end{array}$} & \multicolumn{2}{|c|}{$\mathrm{C}_{\mathrm{f}}\left(\mu \mathrm{g} \mathrm{mL}^{-1}\right)$} & \multicolumn{2}{|c|}{$\mathrm{Q}\left(\mu \mathrm{g} \mathrm{g}^{-1}\right)$} \\
\hline & & & & MIM & NIM & MIM & NIM \\
\hline \multirow{5}{*}{ Tea extraction } & \multirow{5}{*}{200} & EGCG & 18.73 & 11.12 & 15.48 & 1548.85 & 697.20 \\
\hline & & GCG & 2.80 & 2.79 & 2.75 & 10.80 & 11.12 \\
\hline & & ECG & 1.32 & 1.21 & 1.24 & 12.07 & 12.43 \\
\hline & & EGC & 2.67 & 2.10 & 2.17 & 117.72 & 104.50 \\
\hline & & C & 0.17 & 0.12 & 0.13 & 10.14 & 8.76 \\
\hline
\end{tabular}

$\mathrm{C}_{\mathrm{s}}$ : Initial concentration of sample, $\mathrm{C}_{0}$ : concentration of monomer (before adsorption), $\mathrm{C}_{\mathrm{f}}$ : concentration of monomer (after adsorption). 


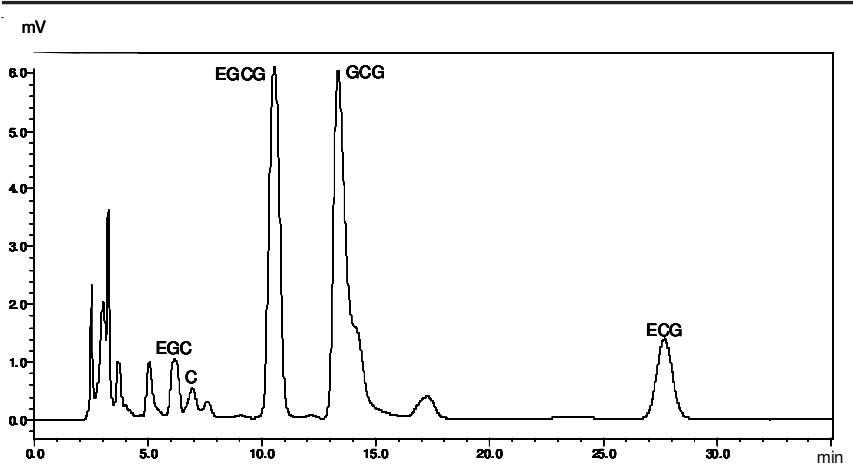

(A)

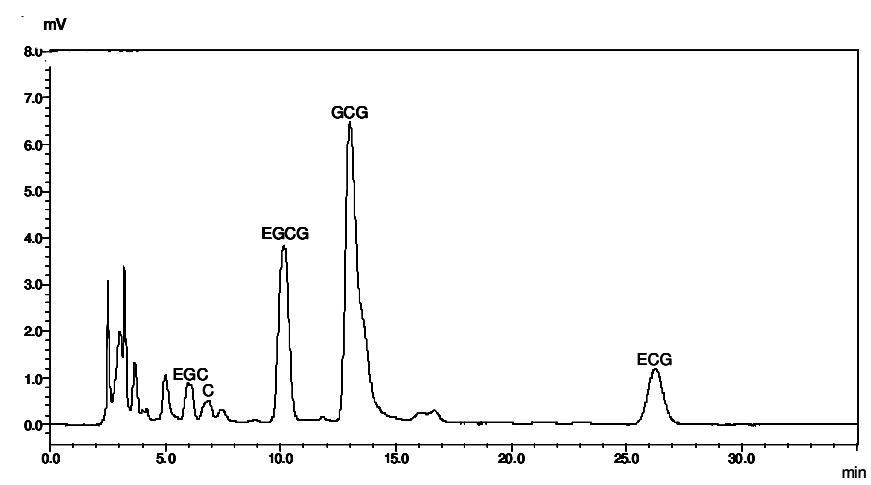

(B)

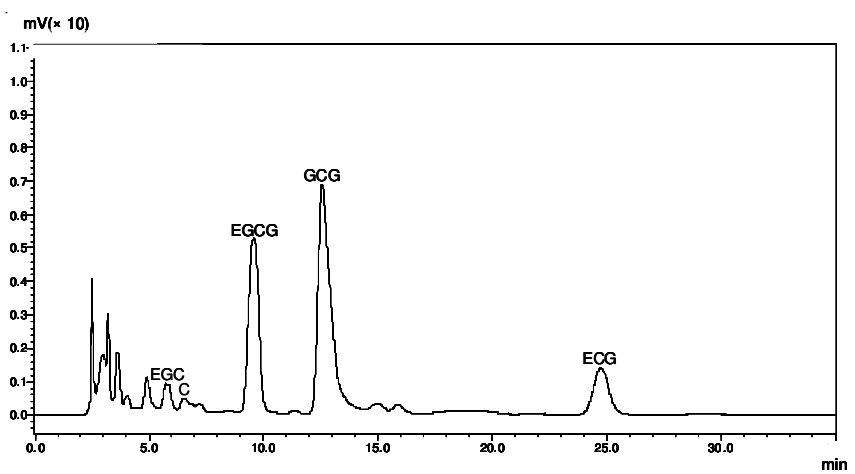

(C)

Fig. 7. MIM and non-molecularly imprinted membrane (NIM) toward the initial extract of tea (A) the initial solution, (B) the solution after adsorbed by MIM, (C) the solution after adsorbed by NIM

The results of the method were calculated and summarized in Table-2. As could be seen, the adsorption capacity of MIM for EGCG reached $1548.85 \mu \mathrm{g} \mathrm{mL} \mathrm{m}^{-1}$ during the initial tea extraction and were 156.23, 146.80, 131.29 and 13.52 times than its for C, GCG, ECG and EGC, respectively. Moreover, the amount between MIM and NIM for template EGCG appeared greater gap $\left(1548.85 \mu \mathrm{g} \mathrm{mL}^{-1} / 697.20 \mu \mathrm{g} \mathrm{mL}^{-1}\right)$ and the amount of MIM was 2.27 more than that of NIM while the gaps for C, GCG, ECG and EGC were 10.14/8.76,10.80/11.12, $12.07 / 12.43,117.72 / 104.50$. By the comparison, it clearly demonstrated good combination and selectivity for the MIM.

\section{Conclusion}

In this paper, MIM of EGCG with high adsorption and selectivity was successfully developed for the first time. The adsorption behavior of the imprinted hybrid membrane fitted the Langmuir model well, suggesting only one kind of binding site existed in the membrane. We concluded that such porous imprinted membranes were very useful for selectivity adsorption of mixture of EGCG and its analogue. However, it was a disadvantage that the MIM was prepared and its characterization of adsorption studied only in organic phase (methanol). In order to make the molecular imprinted membrane applicable in the future, this would be research subject of our subsequent work.

\section{ACKNOWLEDGEMENTS}

The financial support was provided by grants from the National Natural Science Foundation of China (30970309 and 31201456), the China Postdoctoral Science Foundation (20100471379) and the Project Funded by the Priority Academic Program Development of Jiangsu Higher Education Institutions (PAPD).

\section{REFERENCES}

1. S. Bettuzzi, M. Brausi, F. Rizzi, G. Castagnetti, G. Peracchia and A. Corti, Cancer Res., 66, 1234 (2006).

2. S.A. Wiseman, D.A. Balentine and B. Frei, Crit. Rev. Food Sci. Nutr., 37, 693 (1997).

3. N. Salah, N.J. Miller, G. Paganga, L. Tijburg, G.P. Bolwell, C. Rice-Evans, Arch. Biochem. Biophys., 322, 339 (1995).

4. I.E. Dreosti, Nutr. Rev., 54, S51 (1996).

5. Y. Kuroda and Y. Hara, Mutat. Res., 436, 69 (1999).

6. H. Nakane and K. Ono, Biochemistry, 286, 83 (1991).

7. S. Sakanaka, N. Shimura, M. Aizawa, M. Kim and T. Yamamoto, Biochemistry, 56, 592 (1992).

8. L.B.M. Tijburg, T. Mattern, J.D. Folts, U.M. Weisgerber and M.B. Katan, Food Sci. Nutr., 37, 771 (1997).

9. F. Hashimoto, G. I. Nonaka and I. Nishiki, Chem. Pharm. Bull., 37, 77 (1989).

10. L. Yao, Y. Jiang, N. Datta, R. Singanusong, X. Liu, J. Duan, K. Raymont, A. Lisle and Y. Xu, Food Chem., 84, 253 (2004).

11. T.Y. Guo, Y.Q. Xia, J. Wang, M.D. Song and B.H. Zhang, Biomaterials, 26, 5737 (2005).

12. D. Kriz and K. Mosbach, Anal. Chim. Acta, 300, 71 (1995).

13. A.G. Mayes and K. Mosbach, Trends Anal. Chem., 16, 321 (1997).

14. P.K. Owens, L. Karlsson, E.S.M. Lutz, L.I. Andersson, Trends Anal. Chem., 18, 146 (1999).

15. G. Vlatakis, L.I. Andersson, R. Muller, K. Mosbach, Nature, 361, 645 (1993).

16. T.A. Sergeyeva, S.A. Piletsky, A.A. Brovko, L.A. Slinchenko, L.M. Sergeeva, T.L. Panasyuk and A.V. El'skaya, Analyst, 124, 331 (1999).

17. M.C. Hennion, J. Chromatogr. A, 856, 3 (1999).

18. M. Komiyama, T. Takeuchi, T. Mukawa and H. Asanuma, Molecular Imprinting, Wiley-VCH Verlag GmbH \& Co. KGaA (2003).

19. M.N. Han, R. Kane, M. Goto and G. Belfort, Macromolecules, 36, 4472 (2003).

20. X.L. Zhu, Q.D. Su, J.B. Cai, J. Yang and Y. Gao, J. Appl. Polym. Sci., 101, 4468 (2006).

21. R. Kielczynski and M. Bryjak, Sep. Purif. Technol., 41, 231 (2005).

22. S.H. Son and J. Jegal, J. Appl. Polym. Sci., 104, 1866 (2007).

23. C. Cristallini, G. Ciardelli, N. Barbani and P. Giusti, Macromal. Biosci., 4, 31 (2004).

24. F. Liu, C.H. Du, B.K. Zhu and Y.Y. Xu, Polymer, 48, 2910 (2007).

25. N.C. Bing, Z.L. Xu, X.J. Wang, Z.G. Yang and H. Yang, J. Appl. Polym. Sci., 106, 71 (2007).

26. E. Birlik, A. Ersöz, E. Açíkkalp, A. Denizli and R. Say, J. Hazard Mater., 140, 110 (2007).

27. J.M. Wang, C.P. Huang, H.E. Allen, L.R. Takiyama, I. Poesponegoro, H. Poesponegoro and D. Pirestani, Water Environ. Res., 70, 1041 (1998).

28. H. Wu, Y.Y. Zhao, M.C. Nie and Z.Y. Jiang, Sep. Purif. Technol., 68, 97 (2009).

29. W.S.W. Ngah, C.S. Endud and R. Mayanar, React. Funct. Polym., 50, 181 (2002).

30. P.P. Xu, W.Z. Xu, X.J. Zhang and Y.S. Yan, Microchim. Acta, 171, 441 (2010).

31. P. Li, Y.H. Wang, R.Y. Ma and X.L. Zhang, J. Food Eng., 67, 253 (2005). 